\title{
TRANSITION FROM ENERGY DISSIPATIVE PROCESSES TO DISPLACEMENT DISCONTINUITIES DURING CONCRETE FAILURE
}

\author{
S.Y. ALAM ${ }^{\dagger}$ AND A. LOUKILI \\ L'UNAM Université, Institut de Recherche en Génie Civil et Mécanique (GeM), UMR-CNRS 6183, Ecole \\ Centrale de Nantes, Nantes, France \\ †'e-mail: syed-yasir.alam@ec-nantes.fr
}

Key words: energy dissipation rate, fracture; concrete, displacement discontinuities

\begin{abstract}
Fracture in concrete beams is mainly governed by two processes: energy dissipation and discontinuous displacement fields or discrete cracks. In the existing literature, the fracture process in quasi-brittle materials, like concrete, is considered by either one of the two processes. In this study we discuss the simultaneous development of both types of fracture. Energy dissipation and crack openings are analyzed during the fracture development by acoustic emission and digital image correlation. The analysis of local failure processes thus indicates three processes which describes overall failure mechanism: (1) high energy dissipation domain during which energy release rate increases. (2) Continuum - dis-continuum phase where energy dissipation rate attains its maximum value, and (3) dis-continuum phase where energy dissipation rate drops and displacement discontinuities start increasing. A new approach is then presented to model fracture of concrete based on transient and steady-state behavior of a local damaging stress.
\end{abstract}

\section{INTRODUCTION}

The description of failure process is one of the key factors to improve the durability and life time. Failure of concrete is usually assessed by the loss of stiffness and material strength due to damage growth associated with microcracks. The mechanisms observed at the macroscopic scale is the result of local fracture process occurring at the material level. Hillerborg et al. [1] presented a cohesive crack model where mechanical behaviour can be modelled by the local fracture behaviour as described by a characteristic post-peak stresscrack opening softening curve. The model however, neglects the fracture energy dissipation before the peak load. Many experimental studies have been published where efforts are made to identify the fracture mechanisms [2] and characteristic sizeindependent material fracture parameters such as fracture energy [3-6] or relative crack length $[7,8]$. Attention is mostly paid on the understanding of the size and shape of fracture process zone (FPZ) ahead the propagating crack tip and the boundary effects on FPZ [813]. Few experimental studies were focused to analyse the energy dissipation or fracture energy during the fracture process $[14,15]$.

In most experimental studies cited in above, fracture or damage is considered either as the source of energy dissipation mechanisms or as the development of displacement discontinuities. Such analysis produced results in which only one type of damage mechanism is monitored i.e. damage as source of energy dissipations or fracture on the basis of discrete crack openings. None of these experimental studies considered that damage in quasi-brittle materials like concrete is governed by two processes (1) high energy dissipation 
mechanisms during which crack openings may or may not be important and (2) displacement discontinuous phase where energy dissipations may or may not be important. Such analysis can only be performed if two techniques can be simultaneously applied; one characterizing the energy dissipation and the other monitoring the displacement discontinuities.

In this paper acoustic emission (AE) and digital image correlation (DIC) are applied simultaneously on bending tests of notched specimens. AE parameters like events and acoustic energy are analysed to determine energy release rate and the fracture growth due to energy dissipation mechanisms. Full-field displacement are obtained by DIC; where crack openings are measured on the surface of specimen [7,8]. The transition from energy dissipation phase to displacement discontinuity phase is discussed. A new approach is then presented to model the local fracture process based on the transient and steady state response of a local damage parameter. The transient phase indicates the energy dissipation and the steady phase indicates the crack opening phase.

\section{EXPERIMENTAL PROCEDURE}

Two sizes of concrete beams (designated hereafter as $D 1$ and $D 2$ ) with geometrically similar dimensions (length $l$ and depth $D$ ) and one constant dimension (width $B$ ) were tested. The cross sectional depths $D$ were 100 , and $200 \mathrm{~mm}$ respectively with constant width $B$ equals to $100 \mathrm{~mm}$ and the span to depth ratio $(l / D)$ equal to $3: 1$. The beams were notched at mid-span with a notch length $(a)$ varying proportionally to the size of the beam $(a / D$ was kept equal to 0.2). The design of the beam followed the RILEM recommendation [4].

The coarse aggregate used in the concrete mixture had a maximum size of $D_{\text {agg }}$ equals to $10 \mathrm{~mm}$. The average values of axial compressive strength, split tensile strength and the Young's Modulus at 28 days were $58 \mathrm{MPa}$, 5.76 $\mathrm{MPa}$ and $45 \mathrm{GPa}$ respectively. The three point bending tests were performed with a controlled crack mouth opening displacement (CMOD) rate of $0.05 \mu \mathrm{m} / \mathrm{sec}$.
The DIC method was applied and images were continuously obtained during the whole loading branch. Displacement fields were calculated and crack opening were derived. The AE system comprised of a generalpurpose interface bus $(2 \times$ PCI-DISP 4 having 4 channels each). A 3D analysis is performed for the localization of $\mathrm{AE}$ events using 8 piezoelectric transducers. The details of DIC and AE setup can be found in [8].

\section{EXPERIMENTAL RESULTS AND ANALYSIS}

The average mechanical curves are presented in Figure 1 which shows the characteristic quasi-brittle response of concrete material. The behavior is typical and explains the overall load carrying capacity of the concrete beams. Different stages of the failure process of the beams cannot be assessed by this macroscopic response.

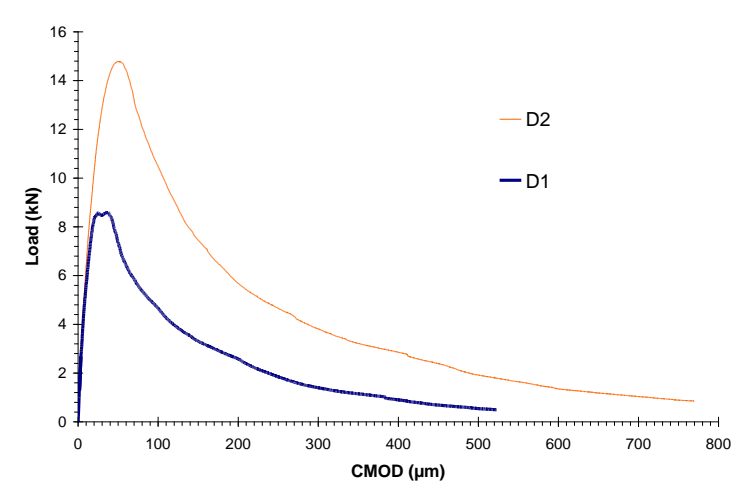

Figure 1: Macroscopic mechanical behavior of beams.

\subsection{Damage growth by acoustic emission activity}

The localization map of $\mathrm{AE}$ sources or events detected during the loading test in concrete beams is presented in Figure 2. Many experimental studies have shown that the scattering zone of AE high intensity sources can characterize the fracture process zone of concrete [11]. The number of AE sources detected and the measured voltage transient can be related to the microcrack volume in the material through a series of deconvolution [16]. The accuracy of AE source location in concrete is affected by several factors, 
including the heterogeneous nature of the material system. Even if a crack is located, the error can be large depending on the size of the tested structure and the distance of the sources to the sensors [17]. The analysis of fracture process may be more reliable on the basis of number of $\mathrm{AE}$ sources as compared to the location of AE sources.

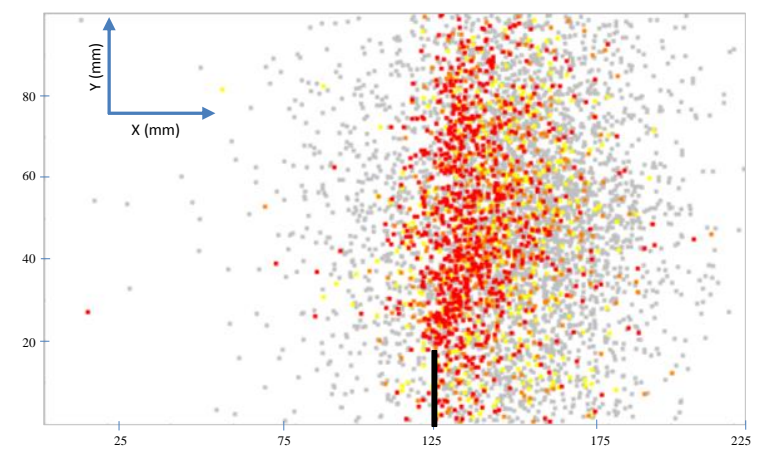

Figure 2: Localization map of AE sources in $D 1$ beam.

As loading increases, the number of $\mathrm{AE}$ events $\left(E_{v}\right)$ increases (Figure 3$)$. The curves show no or low activity in the initial part, during which loadings are elastic. Afterwards, a smooth increase in $E_{v}$ can be observed due to progressive increase in the damage. In order to observe the development of damage and to relate the development of $\mathrm{AE}$ events with failure process, $\mathrm{AE}$ event rate $\left(\underline{E}_{\underline{v}}{ }^{\prime}\right)$ is plotted in Figure 4. It is computed by measuring the increase in the event number in $\mathrm{AE}$ events $\Delta E_{v}$ over a very small interval of CMOD $(\triangle \mathrm{CMOD}$ $\approx 1 \mu \mathrm{m}$ ) as shown in Figure 3 .

$$
E_{v}{ }^{\prime}=\Delta E_{v} / \Delta t
$$

where $\Delta t$ is the corresponding time interval.

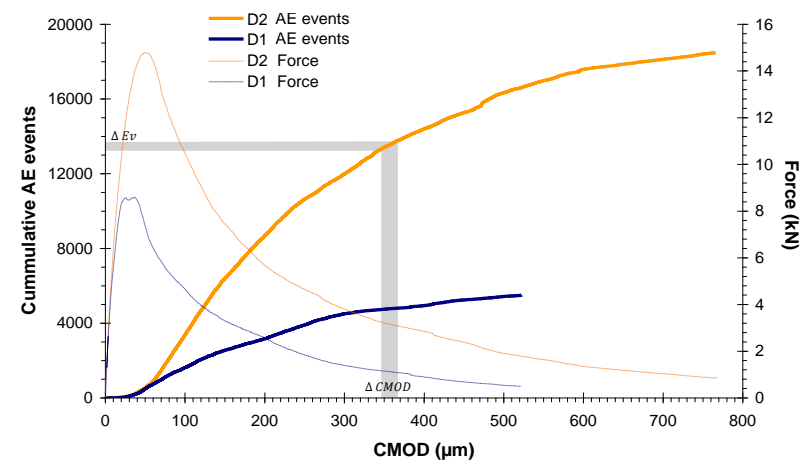

Figure 3: Increase of cumulative AE events during load tests.

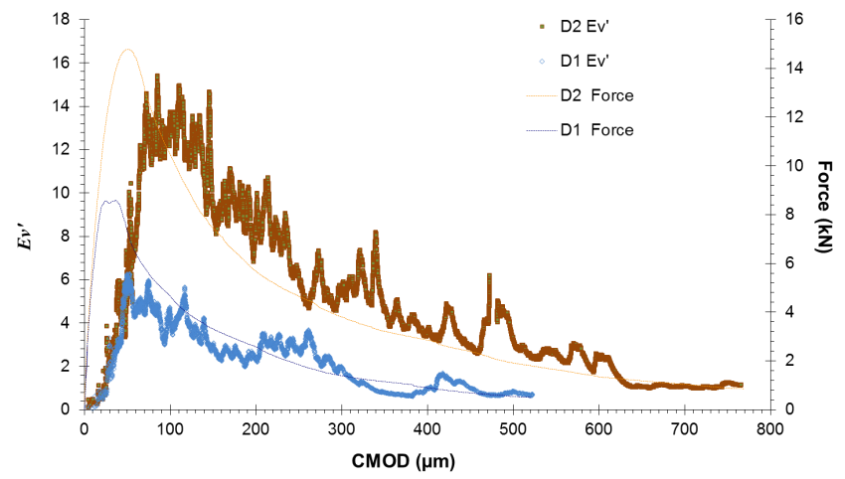

Figure 4: Evolution of AE event rate during the load test.

Thus Figure 4 represents the AE event rate or simply the rate of development of new microcracks during the fracture process of concrete beams. During the initial loadings, $E_{v}{ }^{\prime}$ is very low. Due to progressive microcracking in concrete, $E_{v}{ }^{\prime}$ increases due to increasing rate of microcracks formation. It has been confirmed by several studies that microcracks are being formed in fracture process zone and the size of FPZ increases as the load approaches peak load [8]. It should be noted that $E_{v}{ }^{\prime}$ continues to increase even after peak loading and then it starts decreasing. The decreasing segment of $E_{v}{ }^{\prime}$-CMOD curve for each beam shows the same trend as shown by the post-peak segment of Force-CMOD curve for the corresponding beam.

\subsection{Acoustic emission energy dissipation}

The failure process of concrete involves mechanisms with various energy dissipation levels. The stress waves resulting from these energy dissipative processes are detected by the piezoelectric sensors which convert them into transient electric signals. Generally, the wave form of the signal possesses the characteristics of the dissipative source phenomenon, although the behavior is somehow corrupted and altered due to geometrical parameters and attenuation of signals. In a simplified approach, the energy of the electric signal can be quantified by integrating the square of voltage transient of each channel.

$$
E_{n, i}=\int_{t 0}^{t 1} V_{i}(t)^{2} d t
$$


Such energy dissipative mechanisms release major part of the stored potential energy. The failure criterion in concrete is generally described by the equilibrium condition between energy dissipation during fracture mechanisms and potential energy available for fracture growth. The latter is usually measured by load-displacement curves as in case of Hillerborg fracture energy [3]. The energy accumulated in the electric signal has been used extensively in the literature to study the energy release during fracture growth. The AE energy $E_{n}$ is plotted in Figure 5 . In the pre-peak loadings, $E_{n}$ is very low and it increases smoothly when the load increases. The dissipation process is progressive unlike brittle materials which fail due sudden release of potential energy.

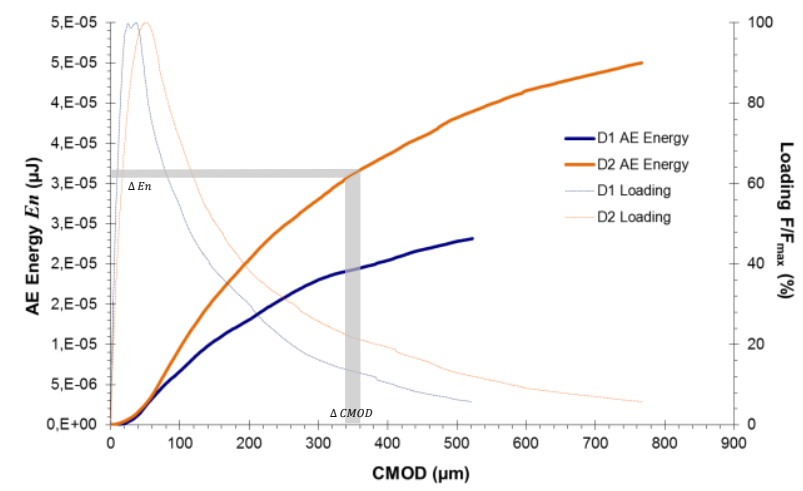

Figure 5: Increase of cumulative AE energy during the load test.

The cumulated AE energy can be related to the fracture energy [15]. Therefore one can relate AE energy release rate with fracture energy release rate or the so called specific fracture energy. Thus rate of AE Energy $\left(E_{n}{ }^{\prime}\right)$ is computed by measuring the increase in the $\mathrm{AE}$ energy $\left(\Delta E_{n}\right)$ over a very small interval of CMOD $(\triangle \mathrm{CMOD}<1 \mu \mathrm{m})$ as shown in Figure 5.

$$
E_{n}{ }^{\prime}=\Delta E_{n} / \Delta t
$$

where $\Delta t$ is the corresponding time interval.

Three distinct stages of development of $E_{n}{ }^{\prime}$ can be noticed in Figure 6. During the first stage, the AE Energy release rate $E_{n}{ }^{\prime}$ increases under the pre-peak loadings. At peak load, $E_{n}{ }^{\prime}$ increases suddenly and it further increases during the initial post-peak loadings. $E_{n}{ }^{\prime}$ becomes maximum at about $85 \%$ post-peak loading. The energy dissipations mechanisms thus become very active near the peak load. These mechanisms are usually centered in the fracture process zone. It should be noticed that energy release rate $E_{n}{ }^{\prime}$ is not maximum at peak load, rather peak load is defined by a very sudden increase in the energy release rate. The maximum value of $E_{n}{ }^{\prime}$ is achieved at about $85 \%$ peak loading.

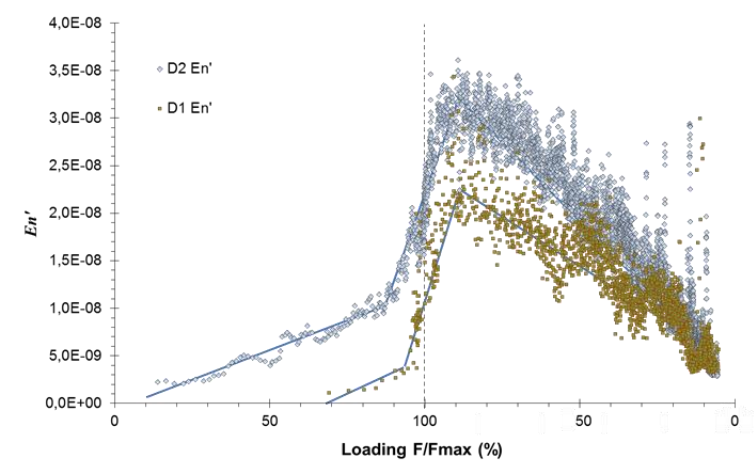

Figure 6: Evolution of AE energy rate with loading steps.

\subsection{Evolution of crack openings}

Figure 7 presents the evolution of crack openings (CODs) with the increase in CMOD. Two phases of crack opening can be noticed. Initially, the crack openings are very small, however, in the second phase a linear relationship can be noticed between COD and CMOD. It indicates that during the microcracking phase, the crack opening is very small and it is difficult to estimate its relationship between intervals of CMOD. After the nucleation of microcracks, or formation of macrocrack, the relationship between crack opening and CMOD is essentially linear. This is verified at different Y locations along the crack. 


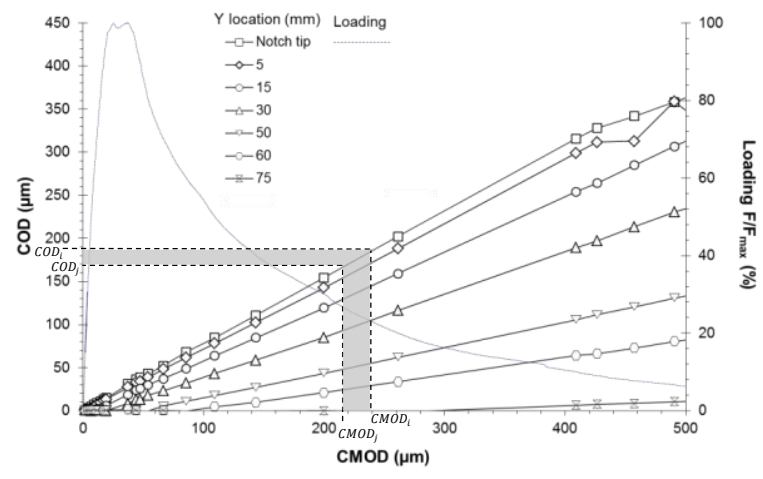

Figure 7: Increase of crack openings during the load test in $D 1$ beam.

The evolution of crack openings with loading intervals is presented in Figure 8. It can be observed that during pre-peak loadings, there are no crack openings. Thus displacement discontinuities are very small and may be in the form of distributed microcracks near the notch tip. Crack opening increases almost linearly with the loadings during the pre-peak loadings. The behavior changes near the peak load and an increase in the crack openings is observed in the postpeak loadings.

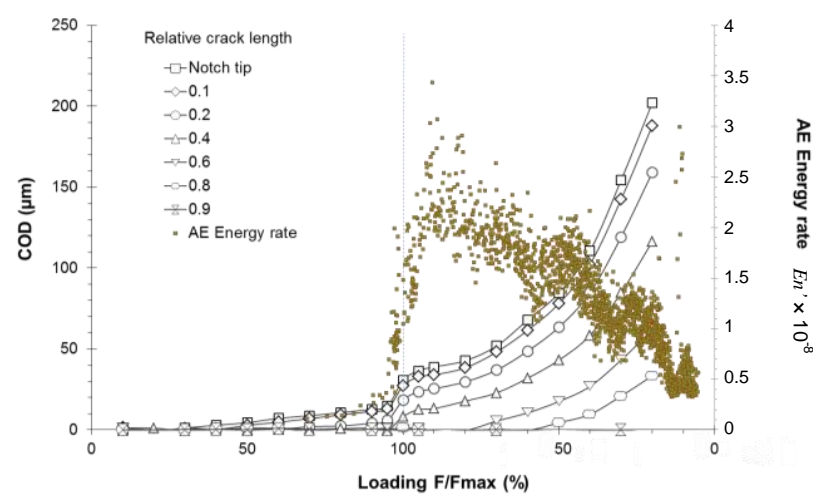

Figure 8: Increase of crack openings with loading steps.

When a mechanical load is applied to a concrete specimen, the initial response of the material can be described by the equilibrium condition between internal forces and external solicitations. As the loading increases, the equilibrium condition is disturbed by other stress relief phenomena and the material begins to fail. Based on the experimental results presented above, the macroscopic failure process can be described by considering three stages: (1) Stage of high energy release rate. It is the first stage of failure process when local crack opening are very small and displacement discontinuities are in the form of very small distributed microcracks. The energy is released due to toughening mechanisms or stress relief processes and material fracture resistance is gradually lost. This phase can be observed e.g. in Figure 6 by increase in the acoustic emission energy rate. (2) The continuum to discontinuum stage. It is a very short phase in which the energy release rate is maximum and the material losses it fracture resistance. During this phase, the bridging or cohesive forces (e.g. due to aggregate restraint or fiber resistance in case fiber reinforced concrete) become active. The material changes from a continuum to a dis-continuum. (3) Displacement discontinuity phase. The material comes to a new local equilibrium condition which can be described by fracture mechanics laws e.g. cohesive stress-crack opening relationship. During this phase as observed in Figure 6 and 8, crack opening follows a non-linear relationship with residual stress.

\subsection{Transient behavior during fracture of concrete}

The complete local fracturing process can be modelled as a transient response and a steady state response of a damping variable. It is the natural response of the material when equilibrium condition is disturbed until the equilibrium is achieved by a new set of conditions. The response is similar to the behavior of stress wave detected in the form of acoustic emission signal from a local fracture source. However, in this case, the transient behavior and the steady state behavior describe the complete behavior of the failure process of the local material point.

The transient response of material can be observed in Figure 9 for $D 1$ (similar behavior is observed in $D 2$ beam). Here the local response of the material is determined by the following function.

$$
\xi=\frac{C O D_{j}}{C M O D_{j}}-\frac{C O D_{i}}{C M O D_{i}}
$$


where $i, j$ are two neighboring loading states as shown in Figure 7. $\triangle C M O D=C M O D_{j}-$ $C M O D_{i}=1 \mu \mathrm{m}$. The functional $\xi$ is plotted at different $Y$ locations along the crack profile as a function of crack opening displacement at the same location. There is an initial transient phase and finally a smooth steady state phase. It should be noticed that $\xi$ at the notch tip is not steady, which means the formation of cracks in not a steady state process. As found in many studies, during this phase, very fine microcracks are developed. A simple explanation of the transient behavior is that when a new microcrack is formed in FPZ, stress relaxation in the surrounding microcracks reduces their openings. This process continues and the fracture process zone size increases. Energy release rate as observed from acoustic emission data increases significantly during this phase. When the fracture process zone size reaches a certain limit and a large amount of energy has been released, the microcracks join to form macrocrack. During this shift phase, AE energy release rate is maximum and the local behavior shifts from energy dissipation process to crack opening process. This can be seen in Figure 9 by the steady state response of local crack opening rate.

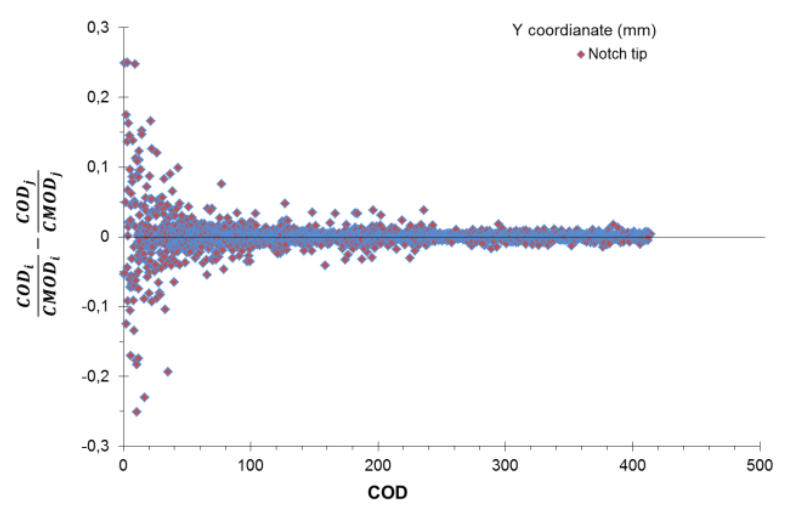

Figure 9: Local damping behavior in D1 beam.

The above analysis of crack openings brings new insight into the fracture process of concrete. The behavior can be generalized into two processes as shown in Figure 10. The crack closing pressure due to cohesive stress acting on the microcracks is an important feature of the fracture process in concrete. In cohesive crack model it is a decreasing function of the crack opening. From Figure 9, 10 , it can be observed that crack closing pressure varies. At some points it is lower than the applied crack opening stress; however, at the other points it is greater than crack opening stress. This can be due to two reasons: (1) the applied stress varies due to stress relaxation during new microcracks formation in the vicinity (2) the crack closing pressure varies due to aggregate restraint, friction between tortuous crack faces and crack branching or crack shielding. The two oppositely acting stresses: applied stress and crack closing stress, become equal after a certain crack opening so called critical crack opening $\left(\mathrm{COD}_{c}\right)$ and material response transits from the transient behavior to the steady-state behavior.

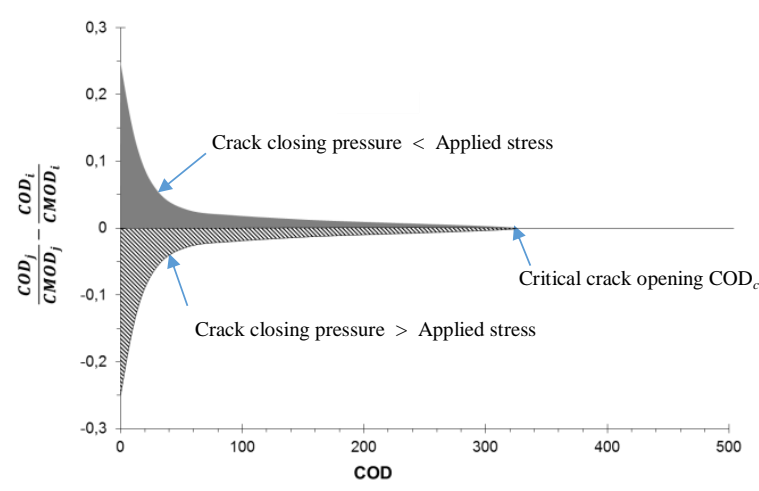

Figure 10: Generalized diagram of the transient behavior observed in figure 9 .

For the two cases observed in figure 10 i.e. when crack closing pressure < applied stress and when crack closing pressure > applied stress, the behavior of crack openings is explained in Figure 11. In the first case Figure 11(a), crack opening increases during to higher applied stress as compared to crack closing pressure and so the parameter $\xi$ is positive. Conversely, in Figure 11(b), crack opening $\left(\mathrm{CMOD}_{j}\right)$ is lower than the crack opening precedent $\left(\mathrm{CMOD}_{i}\right)$ due to higher crack closing pressure as compared applied crack opening stress because of the reasons explained earlier. 


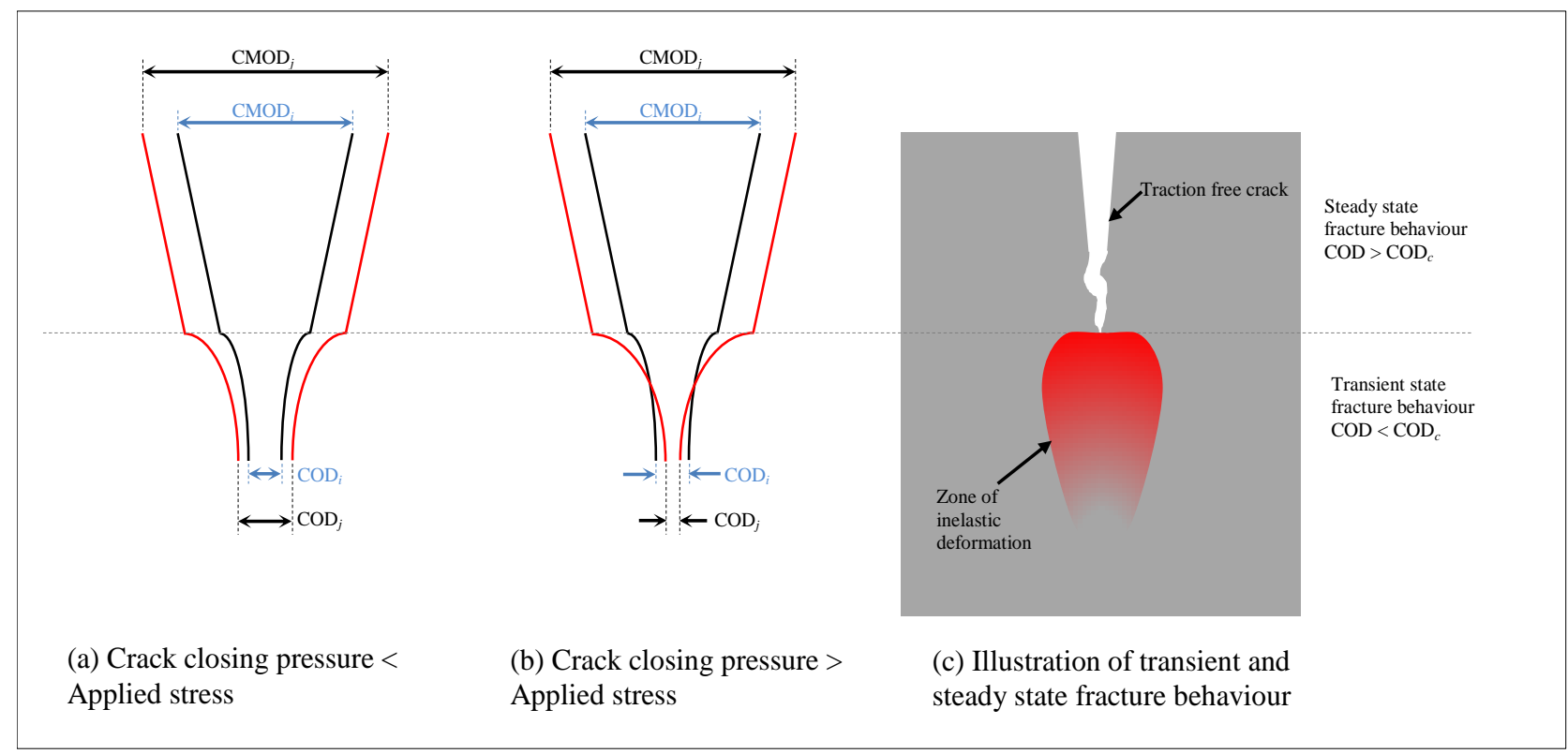

Figure 11: Illustration of crack opening behavior for the two cases observed in figure 10.

The experimental observations and physical interpretations of the fracture process presented in this paper thus define a new way to model the fracture in concrete (Figure 12). It is the based on the relationship between the resulting stress (applied stress - crack closing pressure) and the crack opening. Due the complicated fracturing process; the resulting stress shows a transient response in the beginning which explains the high energy dissipation rate mechanisms. The damping of the resulting stress indicates the rupturing process during which crack opening increases. At certain so called critical crack opening displacement, the relationship between resulting stress and crack opening transits into a steady state behavior which explains the stress free crack opening.

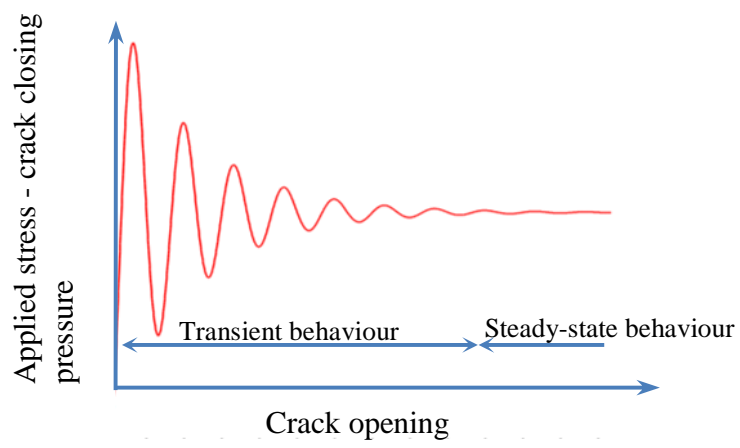

Figure 12: New fracture model based on transient fracture response.

\section{CONCLUSIONS}

The paper describes the transition between energy dissipation phase and dis-continuum phase during the fracture process in concrete. Three point bending tests were conducted; $\mathrm{AE}$ and DIC techniques were applied simultaneously to analyze the energy dissipation process and crack openings respectively. The main conclusions are:

The AE energy release rate indicates three phases of energy dissipation. During the first and second phases, energy release rate increases. The first phase ends just before the peak load; however, the second phase, where the energy release rate reaches the maximum, ends just after the peak load. During these two phases, the rate of microcracks as detected by $\mathrm{AE}$ technique (i.e. only the microcracks releasing AE energy) show a similar behavior. Also during these two phases, crack openings are very small as measured by DIC technique.

- $\quad$ The second phase (or the shift phase) is a very short phase during which AE energy release rate becomes maximum. During this phase, the behavior changes from energy dissipation to crack openings.

- In the third (or the crack opening) phase, the energy release rate begins to decrease with a corresponding increase in crack openings.

A new approach is presented to describe the complete behavior of the local fracture process. The local response of the 
material is represented by a transient behavior of the local resultant stress followed by a steady state response. The transient response indicates the energy dissipation phase; however, the steady state response indicates the crack opening phase.

\section{REFERENCES}

[1] Hillerborg A., Modéer M. and Petersson P.E. 1976. Analysis of crack formation and crack growth in concrete by means of fracture mechanics and finite elements. Cement and Concrete Research 6(6):773781.

[2] Landis E. N. and Bolander J. E. 2009. Explicit representation of physical processes in concrete fracture. Journal of Physics D: Applied Physics 42(21):1-17.

[3] RILEM 50-FMC Recommendation. 1985. Determination of fracture energy of mortar and concrete by means of threepoint bend test on notched beams. Materials and Structures 18:285-290.

[4] RILEM Recommendation. 1990. Sizeeffect method for determining fracture energy and process zone size of concrete. Materials and Structures 23:461-465.

[5] Duan K., Hu X. and Wittmann F. H. 2003. Boundary effect on concrete fracture and non-constant fracture energy distribution. Engineering Fracture Mechanics 70(16):2257-2268.

[6] Karihaloo B. L., Ramachandra Murthy A. and Iyer N. R. 2013. Determination of size-independent specific fracture energy of concrete mixes by the tri-linear model. Cement and Concrete Research 49:82-88.

[7] Alam S. Y., Loukili A. and Grondin F. 2012. Monitoring size effect on crack opening in concrete by digital image correlation. European Journal of Environmental and Civil Engineering 16(7):818-836.

[8] Alam S. Y., Saliba J. and Loukili A. 2014. Fracture examination in concrete through combined digital image correlation and acoustic emission techniques.
Construction and Building Materials 69:232-242.

[9] Cedolin L., Dei Poli S. and Iori I. 1983. Experimental determination of the fracture process zone in concrete. Cement and Concrete Research 13(4):557-567.

[10] Otsuka K. and Date H. 2000. Fracture process zone in concrete tension specimen. Engineering Fracture Mechanics 65:(2-3)111-131.

[11]Haidar K. and Pijaudier-Cabot G., Dubé J. F. and Loukili A. 2005. Correlation between the internal length, the fracture process zone and size effect in model materials. Materials and Structures 38(2):201-210.

[12] Skarżyński Ł. and Tejchman J. 2010. Calculations of fracture process zones on meso-scale in notched concrete beams subjected to three-point bending. European Journal of Mechanics/A Solids 29:746-760.

[13] Alam S. Y., Kotronis P. and Loukili A. 2013. Crack propagation and size effect in concrete using a non-local damage model. Engineering Fracture Mechanics 109:246-261.

[14] Grégoire D., Verdon L., Lefort V., Grassl P., Saliba J., Regoin J.-P., Loukili A. and Pijaudier-Cabot G. 2015. Mesoscale analysis of failure in quasi-brittle materials: comparison between lattice model and acoustic emission data. Int. J. Numer. Anal. Meth. Geomech. 39:16391664.

[15]Landis E. and Baillon L. 2002. Experiments to relate acoustic emission energy to fracture energy of concrete. Journal of Engineering Mechanics 128(6):698-702.

[16]Landis E. 1999. Micro-macro fracture relationships and acoustic emissions in concrete. Construction and Building Materials 13(1-2):65-72.

[17]Grosse C. and Ohtsu M. 2008. Acoustic Emission Testing in Engineering: Basics and Applications. Springer Verlag, Berlin. 